\title{
RELATIVISTIC FIELD EQUATIONS FROM HIGHER-ORDER POLARIZATIONS OF THE POINCARÉ GROUP
}

\author{
Miguel Navarro ${ }^{1,2}$ 丹, Manuel Calixto ${ }^{1,3}$ and Víctor Aldaya $a^{1,4}$
}

1. Instituto Carlos I de Física Teórica y Computacional, Facultad de Ciencias, Universidad de Granada, Campus de Fuentenueva, 18002, Granada, Spain.

2. Instituto de Matemáticas y Física Fundamental, CSIC, Serrano 113123, 28006 Madrid, Spain.

3. Departamento de Física Teórica y del Cosmos. Facultad de Ciencias. Campus de Fuentenueva, 18002. Granada. Spain.

4. IFIC, Centro Mixto Universidad de Valencia-CSIC, Burjassot 46100Valencia, Spain.

\begin{abstract}
The theory of free relativistic fields is shown to arise in a unified manner from higher-order, configuration-space, irreducible representations of the Poincaré group. A de Sitter subalgebra, in the massive case, and a Poincaré subalgebra, in the massless case, of the enveloping algebra of the Poincaré group are the suitable higher-order polarizations. In particular, a simple group-theoretic derivation of the Dirac equation is given.
\end{abstract}

PAC numbers: 03.65.Pm, 03.50.-z, 11.30.Cp

\footnotetext{
*http://www.ugr.es/mnavarro; e-mail: mnavarro@ugr.es

†e-mail: calixto@ugr.es

‡e-mail: valdaya@ugr.es
} 


\section{Introduction}

The theory of relativistic field equations is older than the Special Theory of Relativity. In fact, it was mainly the Poincaré invariance of Maxwell equations for electromagnetism - equations which had been obtained by Maxwell from theoretical considerations upon previous work by Coulomb, Faraday and Ampere - that motivated the shift from the Galileo group to the Poincaré group as the relativity group of Physics. However, it was the quantum revolution of the twenties that actually impelled this subject to acquire the relevance it has gained then since.

The discovery and, in general, the study of the relativistic field equations has followed basically two approaches, Dirac's and Wigner's. In Dirac's approach, the equations are postulated firstly and their invariance under the Poincaré group is "discovered" afterwards. In Wigner's approach, on the contrary, the representations of the Poincaré group are calculated first and then it is shown that the space which supports these representations equals the space of solutions of some (relativistic) field equations. These approaches have, no doubt, been very fruitful. However, both are somewhat unsatisfactory as in neither of the two the relativistic field equations are directly derived from the relativity group and directly in configuration space. As a consequence of this, the actual group-theoretic origin of the Dirac equation remains unclear. Also, group-theoretic treatments of the relativistic field equations have, in general, fail to enter the standard literature on Quantum Field Theory, which is regrettable given the relevance of the subject (a remarkable exception to this rule is Ref. [1]). This and other shortcomings of Wigner's (and Dirac's) approaches may be regarded as symptoms that the theory of relativistic equations is in need of a process of aggiornamento, which, by using modern representation techniques should clarify, simplifly and thus improve it. 
The present paper is meant to be a first step in this process of aggiornamento. We show how the relativistic field equations can be obtained in configuration space and directly from the relativity group - as providing linear, finite-component, irreducible representations of the Poincaré group. We basically use standard techniques of the theory of quantization on a coset space $G / H$, but generalize them so as to fetch some higher-order polarization techniques from the Group Approach to Quantization (GAQ) formalism (see Ref. [2] and references therein). These higher-order polarization conditions, which are being laid on solid grounds within the GAQ formalism [2, 3, 4] generalize both the familiar Casimir-operator conditions and the formalism of induced representation, which uses first-order polarizations only.

The main goal of the present paper is to show how these higher-order quantizations give rise to the right configuration-space equations of motion for the classical fields. In this way, we provide a direct group-theoretic construction of these equations, in particular Dirac equation. As far as we know, no derivation similar to ours has been presented before.

The structure of the present paper is as follows. The mathematical foundations of our approach are presented in Sect. 2 and are applied in Sect. 3 to the Poincaré group. In Sect. 4, we touch upon several facts which may be useful to derive maximum benefit from our study. The Appendix, which contents some general features of the Clifford and Kemmel algebras, has been added to facilitate the reading of the present paper.

Excellent complementary discussions on the subject as well as more references to original works can be found in Refs. [1] and [5]-[1].

\section{Higher-order polarizations}

Our developments are based on a result which has recently been proven in the context of GAQ (see Ref. [2]). A version of this statement, suitably 
taylored for the subject at hand, can be presented in the following form:

Let $G$ be a connected and simply connected Lie group and $\mathcal{U}^{L}(G)$ its universal left-enveloping algebra. Let $H$ be an Abelian subgroup of $G$ and $\mathcal{A}$ a maximal subalgebra of $\mathcal{U}^{L}(G)$ which does not include the Lie algebra of $H$. Then, $G$ is pseudo-irreducibly represented on the functions $\Psi: G \longrightarrow C$, which fulfil

$$
Y . \Psi=0, \quad \forall Y \in \mathcal{A}
$$

Pseudo-irreducibility means here that the Hilbert space is such that any differential or pseudodifferential operator commuting with the representation is a multiple of the identity, but it may possibly contain invariant subspaces which can (only) be distinguished under the action of some non pseudodifferential operators which are external to the group.

In other words, the theorem says that by imposing a sufficient number of (first- or higher-order) left equations of motion we arrive at a representation of the group which is basically irreducible. If there appear non pseudodifferential operators which mix subspaces which are invariant under the group, they can be taken care of, case by case, at the end of the procedure (for more details and examples see Ref. [2]).

This theorem can be extended - by means of a corollary which is presented next and which can be proved through similar steps to those of the theorem above - to the case we consider in the present paper, in which the left-subalgebra $\mathcal{A}$ is finitely generated and is non-trivially represented. A definition will prove useful:

Definition: We shall say that $\mathcal{B} \subset \mathcal{U}^{L}(G)$ is a weak subalgebra if it closes with structure constants which may involve Casimir operators. In other words, $\mathcal{B}$ closes as a subalgebra if the Casimir operators are considered to be numbers. Corollary: Under the same hypothesis of the theorem, consider now that $\mathcal{A}$ is generated by the Casimir operators $C_{a}, a=1, \ldots k$, and a weak subalgebra 
$\mathcal{B}$ which is finite-dimensional with basis $\left\{Y_{i}\right\}_{(i=1, \ldots n)}$. Let $V$ be a finite-

dimensional space where $\left\{Y_{i}^{L}\right\}$ is irreducibly represented by $\left\{\alpha_{i}\right\}$, where $\alpha_{i}$ are finite-dimensional matrices. Let $\mathcal{H}$ be the space of functions $\Psi: G \longrightarrow V$ such that

$$
\begin{aligned}
C_{a} \cdot \Psi & =c_{a} \Psi, \quad a=1, \ldots k \\
Y_{i}^{L} \cdot \Psi & =\alpha_{i} \Psi, \quad i=1, \ldots n
\end{aligned}
$$

for some numbers $c_{a}$. Then $G$ is pseudo-irreducibly represented on $\mathcal{H}$.

The theorem and its corollary does not directly apply to the Poincaré group, as it is not connected. However, in this case, the discrete symmetries -parity $P$, time reversal $T$ and the product $P T$ - which cause the group not to be connected, and which behave much in the same way as the abovementioned non pseudo-differential operators which are external to the group, turn out to be simple to manage. Let us assume we have been able to find out the finite-component irreducible representations of the connected part of the Poincaré group, which the theorem above shows us how to achieve. Then, a discrete symmetry $I$ either preserves a given representation $D$ or changes it to another representation $D^{*}$. In any case, since $I^{2}=1_{\mathcal{P}}$, we must have, save for a factor, $D^{* *}=D$ and any discrete symmetry mixes two representations at most. Therefore, the irreducible representations of the complete group prove to be a discrete sum of representations of its connected-to-the-identity component. More detailed discussions can be found in Ref. [6, 8, [1].

\section{The Poincaré group and relativistic wave equations}

The Poincaré group $\mathcal{P}$ is the semidirect product of the Lorentz group $\mathcal{L}$ and the group of translations $\mathcal{T}$ : 


$$
\begin{aligned}
& \Lambda^{\prime \prime}=\Lambda^{\prime} \Lambda \\
& a^{\prime \prime}=a^{\prime}+\Lambda^{\prime} a
\end{aligned}
$$

With a convenient parameterization of the group, the left- and right-invariant vector fields takes the form (see Refs. [12, [13]):

$$
\begin{aligned}
J_{\mu \nu}^{L} \equiv X_{\epsilon_{\mu \nu}}^{L} & ={ }^{L} T_{\mu \nu}^{\alpha \beta} \frac{\partial}{\partial \epsilon^{\alpha \beta}} \\
P_{\mu}^{L}=X_{a_{\mu}}^{L} & =\Lambda_{\alpha \mu} \frac{\partial}{\partial a_{\alpha}}
\end{aligned}
$$

and

$$
\begin{aligned}
J_{\mu \nu}^{R} \equiv X_{\epsilon_{\mu \nu}}^{L} & ={ }^{R} T_{\mu \nu}^{\alpha \beta} \frac{\partial}{\partial \epsilon^{\alpha \beta}}+\left(\delta_{\mu}^{\alpha} \delta_{\nu}^{\beta}-\delta_{\mu}^{\beta} \delta_{\nu}^{\alpha}\right) a_{\beta} \frac{\partial}{\partial a^{\alpha}} \\
P_{\mu}^{R}=X_{a_{\mu}}^{R} & =\frac{\partial}{\partial a_{\mu}}
\end{aligned}
$$

where ${ }^{L} T_{\mu \nu}^{\alpha \beta}$ and ${ }^{R} T_{\mu \nu}^{\alpha \beta}$ are functions of the parameters $\epsilon^{\alpha \beta}$ of the group the actual expression of which we shall not need (the interested reader is referred, nonetheless, to Refs. [13, [12]).

These vector fields close the familiar algebra:

$$
\begin{aligned}
{\left[P_{\mu}^{L}, P_{\nu}^{L}\right] } & =0, \quad\left[J_{\mu \nu}^{L}, P_{\rho}^{L}\right]=\eta_{\nu \rho} P_{\mu}^{L}-\eta_{\mu \rho} P_{\nu}^{L} \\
{\left[J_{\mu \nu}^{L}, J_{\alpha \beta}^{L}\right] } & =\eta_{\nu \alpha} J_{\mu \beta}^{L}-\eta_{\mu \alpha} J_{\nu \beta}^{L}+\eta_{\mu \beta} J_{\nu \alpha}^{L}-\eta_{\nu \beta} J_{\mu \alpha}^{L}
\end{aligned}
$$

We have $\mathcal{T}=\mathcal{P} / \mathcal{L}$ and the Minkowski space can be identified with $\mathcal{T}$. Therefore, we may obtain fields in configuration space by quantizing the Poincaré group with the Lorentz $(\sim S L(2, C))$ subgroup as (part of) the polarization. 
Let us consider, therefore, a representation $S$ of $S L(2, C)$ which is defined on a finite-dimensional vector space $V$. For any function $\Psi: \mathcal{P} \longrightarrow V$, $\Psi=\Psi(\Lambda, a)$, the Lorentz polarization condition takes the form:

$$
\Psi\left(\Lambda \Lambda^{\prime}, a\right)=S\left(\Lambda^{\prime-1}\right) \Psi(\Lambda, a)
$$

By taking $\Lambda^{\prime}=\Lambda^{-1}$, we obtain:

$$
\Psi(\Lambda, a)=S^{-1}(\Lambda) \Psi(I, a) \equiv S^{-1}(\Lambda) \Phi(a)
$$

Therefore, as a desired result of our procedure, we have obtained functions which are defined solely over Minkowsky space. Moreover, Eqs. (8-9) and the natural (left) action of the group on the functions $\Psi$ imply that the Poincaré group acts on the physical fields $\Phi$ as desired:

$$
((\Lambda, a) \Phi)(x)=S(\Lambda) \Phi\left(\Lambda^{-1}(x-a)\right)
$$

Eq. (8) means that the left-invariant "angular-momentum" vector fields $J_{\mu \nu}^{L}$ have been represented by a finite-dimensional matrix $-S_{\mu \nu}$

$$
J_{\mu \nu}^{L} \rightarrow-S_{\mu \nu}
$$

where

$$
S(\Lambda)=\exp \left\{\frac{1}{2} \epsilon^{\mu \nu} S_{\mu \nu}\right\}
$$

On the other hand, Eq. (10) means that the physical "angular-momentum" operators $J_{\mu \nu} \equiv J_{\mu \nu}^{R}$ are given by the familiar expression:

$$
J_{\mu \nu}=-\left(a_{\mu} \partial_{\nu}-a_{\nu} \partial_{\mu}\right)+S_{\mu \nu}
$$

For the scalar field, we have $S_{\mu \nu}=0$ and for the Proca fields $\left(S_{\mu \nu}\right)_{\alpha \beta}$ $\equiv\left(\Sigma_{\mu \nu}\right)_{\alpha \beta}=\left(g_{\mu \alpha} g_{\nu \beta}-g_{\mu \beta} g_{\nu \alpha}\right)$. For the Dirac field, the $4 \times 4$ matrices 
$S_{\mu \nu} \equiv \sigma_{\mu \nu}$ provide a direct sum of the $\left(\frac{1}{2}, 0\right)$ and $\left(0, \frac{1}{2}\right)$ representations of the restricted Lorentz group. Since parity transforms these representations into each other, the direct sum is necessary to provide an irreducible representation of the complete Lorentz group. When invariance under parity is not required, which is the case of neutrino fields, two-component spinors can be used.

\subsection{The de Sitter higher-order subalgebra}

The condition (\$) is not strong enough to provide irreducible representations of the Poincaré group. In general, reducing the representation will require imposing higher-order polarization conditions [3, 2, 4].

It is clear that any (higher-order) polarization must contain the Casimir operators of the Poincaré group, $P^{2}=\eta^{\mu \nu} P_{\mu}^{L} P_{\nu}^{L}$ and $W^{2}=\eta^{\mu \nu} W_{\mu}^{L} W_{\nu}^{L}$, with $W_{\mu}=\frac{1}{2} \epsilon_{\mu \alpha \beta \nu} J^{\alpha \beta} P^{\nu}$. Therefore, the functions $\Phi(x)$, if supporting an irreducible representation of the Poincaré group, must verify

$$
\begin{aligned}
P^{2} \Phi(x) \equiv \square \Phi(x) & =-m^{2} \Phi(x) \\
W^{2} \Phi(x) \equiv\left(-\frac{1}{2} \square S^{\mu \nu} S_{\mu \nu}+S^{\mu \alpha} S_{\mu \beta} \partial_{\alpha} \partial^{\beta}\right) \Phi & =-m^{2} s(s+1) \Phi(x)
\end{aligned}
$$

where $\partial_{\alpha}=\partial / \partial a^{\alpha}$ and so on and $\square=\eta^{\mu \nu} \partial_{\mu} \partial_{\nu}$. The constant $m$, which we take to be real and non-negative, is the mass of the (quanta of) the field. The constant $s$, which we take to be discrete (positive integer or half integer) is the spin of (the quanta) of the field.

Let us now consider the following operators of the enveloping algebra of the Poincaré group

$$
{ }^{\lambda} X_{\mu}=\lambda P_{\mu}+P^{\rho} J_{\rho \nu}
$$

where the unspecified real number $\lambda$ will be determined later. These secondorder operators ${ }^{\lambda} X_{\mu}$, together with $J_{\mu \nu}$, weakly close a de Sitter (sub-)algebra: 


$$
\begin{aligned}
{\left[{ }^{\lambda} X_{\mu}^{L},{ }^{\lambda} X_{\nu}^{L}\right] } & =J_{\mu \nu}^{L} P^{2} \\
{\left[J_{\mu \nu}^{L},{ }^{\lambda} X_{\rho}^{L}\right] } & =\eta_{\nu \rho}{ }^{\lambda} X_{\mu}^{L}-\eta_{\mu \rho}{ }^{\lambda} X_{\nu}^{L}
\end{aligned}
$$

Therefore, the corollary in Sect. 2 applies here with $H=\mathcal{T}$. Thus, we may get an irreducible representation of the Poincaré group over fields on Minkowski space by imposing the de Sitter algebra $\left\langle J_{\mu \nu}^{L},{ }^{\lambda} X_{\nu}^{L}>\right.$, together with $P^{2}$ and $W^{2}$, as the (higher-order) polarization.

We impose (the rest of) the de Sitter polarization conditions as follows:

$$
\left({ }^{\lambda} X_{\mu}^{L} \Psi\right)^{\alpha} \equiv\left(\left(\lambda P_{\mu}^{L}+P^{L^{\rho}} J_{\rho \nu}^{L}\right) \Psi\right)^{\alpha}=i m\left(\rho_{\mu}\right)_{\beta}^{\alpha} \Psi^{\beta}
$$

where $\rho_{\mu}$ are a set of matrices which must provide a finite-dimensional representation of the de Sitter algebra. In particular, we must have

$$
\begin{aligned}
{\left[\rho_{\mu}, \rho_{\nu}\right] } & =S_{\mu \nu} \\
{\left[S_{\mu \nu}, \rho_{\rho}\right] } & =\eta_{\nu \rho} \rho_{\mu}-\eta_{\mu \rho} \rho_{\nu}
\end{aligned}
$$

The last equation implies

$$
S(\Lambda) \rho^{\mu} S(\Lambda)^{-1}=\Lambda_{\nu}^{\mu} \rho^{\nu}
$$

which, together with Eqs. (18) and (9), implies that the wave functions $\Phi$ obey the equations:

$$
\left(\lambda \frac{\partial}{\partial a^{\mu}}+S_{\mu \nu} \frac{\partial}{\partial a_{\nu}}\right) \Phi(a)=i m \rho_{\mu} \Phi(a)
$$

Since $P^{\mu \lambda} X_{\mu}=\lambda P^{2}$, Eq. (22) yields

$$
\left(i \rho^{\alpha} \frac{\partial}{\partial a^{\alpha}}+\lambda m\right) \Phi=0
$$


Equations of motion of this form, with matrices $\rho^{\mu}$ fulfilling Eqs. (19,20) have been extensively studied in the literature. In particular, it was shown [14] that, for arbitrary spin $s$, eq. (23) leads to an equation of the form:

$$
\left(s^{2} \square+\lambda^{2} m^{2}\right)\left((s-1)^{2} \square+\lambda^{2} m^{2}\right) \cdots \Phi=0
$$

where the last factor is $\left(\lambda^{2} m^{2}\right)$ for integer spin and $\left(\left(\frac{1}{2}\right)^{2} \square+\lambda^{2} m^{2}\right)$ for halfinteger spin. Therefore, for the special fields -scalar, Proca and Dirac- $\lambda$ is fixed (save for an irrelevant sign) whereas, for greater spins, it can have different values:

$$
\lambda= \pm s, \quad \pm(s-1), \ldots \pm 1(s \text { integer }) \quad \pm \frac{1}{2}(s \text { half integer })
$$

This can also be seen by realizing, from Eq. (23), that $\lambda$ must be an eigenvalue of $\rho^{0}$, which has the same eigenvalues as the spin matrices $i S_{i j}, i, j=$ $1,2,3$.

In fact, it is easy to show that for $\lambda=s=\frac{1}{2}$ Eqs. (22) and (23) are equivalent to each other and describe the Dirac particle. The matrices $\rho^{\mu}$ prove to be $\rho^{\mu}=\frac{1}{2} \gamma^{\mu}$, with $\gamma^{\mu}$ (a representation of) the Dirac matrices. Also, for $\lambda=s=1$, Eqs. (22) and (23) are equivalent to each other and describe the Duffin-Kemmer-Petiau field, which, depending on the representation taken for the matrices $\rho^{\mu} \equiv \beta^{\mu}$-five dimensional or ten dimensional-is equivalent to the scalar field or the Proca field, respectively (see Appendix).

\section{Massless fields}

In the case of massless fields, for which $m^{2}=0$, the operators $J_{\mu \nu}$ and ${ }^{\lambda} X_{\rho}$ generate a Poincaré algebra. Therefore, in this case the polarization condition to be imposed along with Eq. (8) is:

$$
{ }^{\lambda} X_{\rho} \Psi \equiv\left(\left(\lambda P_{\mu}^{L}+P^{L^{\rho}} J_{\rho \nu}^{L}\right) \Psi\right)^{\alpha}=0
$$


which leads to

$$
\left(\lambda \frac{\partial}{\partial a^{\mu}}+S_{\mu \nu} \frac{\partial}{\partial a_{\nu}}\right) \Phi(a)=0
$$

Here, we do not need to introduce the $\rho^{\mu}$ matrices, and no equation similar to (23) is reached. As $\lambda$ is an eigenvalue of $S^{0 i}$ - for instance $S^{03}$ - we obtain a result similar to that for massive fields: $\lambda$ is fixed for the special fields, and equals the helicity, whereas it can have different values for higher-spin fields. In fact, for $\lambda=1$, Eq. (27) reproduces, depending on the representation for $S_{\mu \nu}$, the wave equation for the scalar or Maxwell fields [15]. For $\lambda=\frac{1}{2}$, Eq.

(27) reproduces, after projecting over the chirality eigenfields with $\left(1 \pm \gamma^{5}\right)$, the equation of motion for neutrinos.

\section{Discussion}

Our analysis is not meant to be exhaustive but rather to show how modern representation techniques serve to give the subject of relativistic field equations a new look. However, we have been able to give a broad if brief view on that subject. Although our emphasis has been placed on the special fields - those with $s \leq 1$, which are by far the most relevant ones, as no elementary particle has yet been found with $s>1$ - the possibility is open to make a more detailed analysis of the higher-spin fields within this formalism. Another interesting extension of the present analysis, which is already under way [19], is towards analysing other relativity groups such as the de Sitter-like groups, which describe fields in curved space.

Finally, let us briefly discuss the unitarity of the representations we have obtained. Since our wave functions depend only on the coordinates of Minkowski spacetime, and their value on different times can be obtained from the Cauchy data and the equations of motions, any invariant scalar product must be of the form 


$$
<\Phi^{\prime} \mid \Phi>=\int_{\Sigma} \mathrm{d} \sigma_{\mu} J^{\mu}\left(\Phi^{\prime}, \Phi\right)
$$

where $\Sigma$ is an space-like Cauchy hypersurface and $J^{\mu}$ a bilinear current, $J^{\mu}=J^{\mu}\left(\Phi^{\prime}, \Phi\right)=\left(J^{\mu}\left(\Phi, \Phi^{\prime}\right)\right)^{*}$, which, in order to give rise to a (space-)timeindependent scalar product, must be divergenceless, $\partial_{\mu} J^{\mu}=0$.

Currents which fulfill these requirements are the following ones [16]

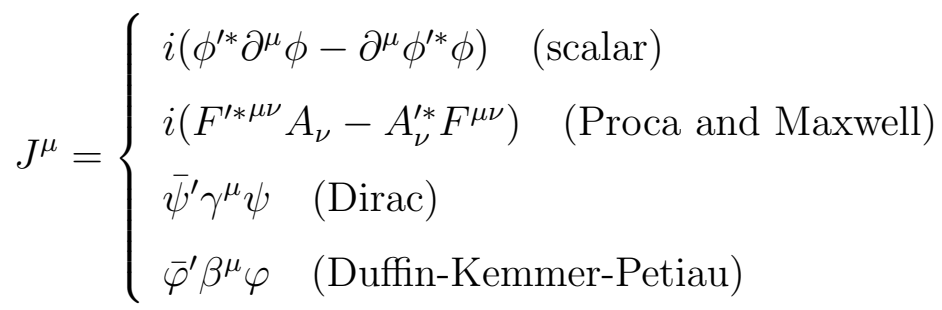

Except for the Dirac field, these products fail to be positive definite. In general, the representations of the complete Poincaré group which are induced from a finite-component representation of its Lorentz subgroup fail to have either a positive definite invariant product (they are not unitary henceforth) or a Hamiltonian which is bounded from below. The Dirac field, for instance, is endowed with a well-behaved scalar product but fails to have a bounded Hamiltonian. The other fields present the reverse behaviour: a positive Hamiltonian but a non-definite "scalar" product. For the Dirac field, the situation is reversed by means of its "grassmannization": the Hamiltonian is made positive whereas the scalar product becomes indefinite. The product is then interpreted, by analogy with other fields, as the electric charge of the (configuration or state of) the field. For one or the other reason, none of these wave equations gives rise to a well-behaved (single-particle) quantum theory. The procedure of second quantization is thus required (For a more detailed view of the procedure of "second-quantization" from a group-theoretic standpoint see Refs. [12, 17, 18, 19]). 


\section{Appendix: The Clifford and Kemmer algebras and the de Sitter group}

Let $\eta_{\mu \nu}$ be a D-dimensional pseudo-euclidean flat metric. Let us assume we are able to find matrices $\gamma_{\mu}, \mu=1, \ldots, D$ such that

$$
\left\{\gamma_{\mu}, \gamma_{\nu}\right\}=\gamma_{\mu} \gamma_{\nu}+\gamma_{\nu} \gamma_{\mu}=2 \eta_{\mu \nu}
$$

and let $\rho_{\mu} \equiv \frac{1}{2} \gamma_{\mu}$ and

$$
S_{\mu \nu}=\left[\rho_{\mu}, \rho_{\nu}\right] \equiv \rho_{\mu} \rho_{\nu}-\rho_{\nu} \rho_{\mu}=\frac{1}{4}\left[\gamma_{\mu}, \gamma_{\nu}\right]
$$

then the matrices $\rho_{\mu}$ and $S_{\mu \nu}$ close a de Sitter algebra:

$$
\begin{aligned}
{\left[S_{\mu \nu}, S_{\alpha \beta}\right] } & =\eta_{\nu \alpha} S_{\mu \beta}--\eta_{\mu \alpha} S_{\nu \beta}+\eta_{\mu \beta} S_{\nu \alpha}-\eta_{\nu \beta} S_{\mu \alpha} \\
{\left[S_{\mu \nu}, \rho_{\rho}\right] } & =\eta_{\nu \rho} \rho_{\mu}-\eta_{\mu \rho} \rho_{\nu} \\
{\left[\rho_{\mu}, \rho_{\nu}\right] } & =S_{\mu \nu}
\end{aligned}
$$

which follows from the following identity:

$$
[[A, B], C]=\{A,\{B, C\}\}-\{B,\{A, C\}\}
$$

Therefore, if we are given a representation of the Clifford algebra (30), we automatically obtain a representation of the de Sitter group.

As is well known, for $D$ even, there is only one irreducible representation of the Clifford algebra whereas, for D odd, there are two irreducible unitarilyinequivalent representations which nevertheless differ only by a sign. In four dimensions, the Clifford algebra is irreducibly represented by the Dirac matrices. In three dimensions, it is represented by the Pauly matrices $\sigma_{i}$. The fact that $\left[\sigma_{i}, \sigma_{j}\right]=\epsilon_{i j k} \sigma_{k}$ provides direct proof of the well-known result that the (pseudo-)orthogonal algebras in four dimensions are equivalent to a direct 
sum of two $A_{1}$ algebras. In two dimensions the Clifford algebra is represented by $\left\{\sigma^{1}, \sigma^{2}\right\}$, which close a $A_{1}$ algebra.

The Kemmer algebra is defined by a set of matrices $\beta^{\mu}, \mu=1, \ldots D$, which obey the relations

$$
\beta^{\mu} \beta^{\lambda} \beta^{\nu}+\beta^{\nu} \beta^{\lambda} \beta^{\mu}=\eta^{\mu \lambda} \beta^{\nu}+\eta^{\nu \lambda} \beta^{\mu}
$$

We now define the matrices $S_{\mu \nu}$ as follows:

$$
S_{\mu \nu}=\left[\beta_{\mu}, \beta_{\nu}\right]
$$

Then, by using the relation (34) and the Jacobi identity, it is easy to show that the matrices $\beta_{\mu}$ and $S_{\mu \nu}$ also close a de Sitter algebra.

In 4 dimensions, the Kemmer algebra has, apart from the trivial one, two irreducible representations: a five-dimensional one, which corresponds to the scalar field and its four spacetime derivatives, and a ten-dimensional other which corresponds to the four components of a vector field and the six components of its associated stress tensor.

Acknowledgements: M. N. is grateful to the Spanish M.E.C., C.S.I.C. and I.M.A.F.F. for a research contract, and to the Imperial College, where this paper was written, for its boundless hospitality. M. Calixto acknowledges the Spanish M.E.C for a F.P.I. grant.

This work was partially supported by the DGICYT. 


\section{References}

[1] S. Weinberg, "The quantum theory of fields", Cambridge: Cambridge University Press, 1996. Chapters 2 and 5.

[2] V. Aldaya, J. Guerrero and G. Marmo, "Quantization on a Lie group: higher-order polarizations", contribution to Symmetry and Science X, Bregenz (Austria) 1997 (to appear in the proceedings), hep-th/9710002.

[3] V. Aldaya, J. Bisquert, R. Loll and J. Navarro-Salas, J. Math. Phys. 33, 3087 (1992).

[4] V. Aldaya and J. Navarro-Salas, Comm. Math. Phys. 139, 433 (1991).

[5] E.M. Corson, "Introduction to tensors, spinors and relativistic wave equations: (relation structure)", Blackie, London 1953.

[6] L. Fonda and G.C. Ghirardi, "Symmetry principles in quantum physics", Marcel Dekker, New York, 1970.

[7] W. Greiner, "Relativistic quantum mechanics: wave equations", Springer-Verlag, 1990.

[8] M.A. Naimark, "Linear representations of the Lorentz group", Pergamon Press, London 1964.

[9] Y. Takahashi, "An introduction to field quantization", Pergamon Press, 1969.

[10] H. Umezawa, "Quantum Field Theory", North-Holland Publishing Co., Amsterdam, 1956.

[11] G. Velo and A.S. Wightman, "Invariant wave equations", SpringerVerlag, Berlin, 1978.

[12] V. Aldaya, J.A. de Azcarraga and S. García, J. Phys. A21, 4265 (1988) 
[13] V. Aldaya and J.A. de Azcarraga, Ann. Phys. N.Y. 165, 484 (1985).

[14] H.J. Bhabha, Rev. Mod. Phys. 17, 200 (1945); 21, 451 (1949).

[15] Harish-Chandra, Proc. Roy. Soc. A186, 502 (1946).

[16] M. Navarro, V. Aldaya and M. Calixto, J. Math. Phys. 37, 206 (1996), hep-th/9501085.

[17] M. Navarro, V. Aldaya and M. Calixto, J. Math. Phys. 38, 1454 (1997), hep-th/9612068.

[18] V. Aldaya, M. Calixto and M. Navarro. "The electromagnetic and Proca fields revisited: a unified quantization', Int. J. Mod. Phys. A12, 3609 (1997), hep-th/9609083.

[19] M. Calixto, V. Aldaya and M. Navarro, "Quantum field theory in curved space from a second quantization on a group", J. Math. Phys. (Submitted), hep-th/9701180. 